\title{
On the Footprints of Join Points: The Blueprint Approach
}

\author{
Walter Cazzola \\ DICo - Department of Informatics and Communication, \\ Università degli Studi di Milano \\ cazzola@dico.unimi.it \\ Sonia Pini \\ DISI - Department of Informatics and Computer Science, \\ Università degli Studi di Genova \\ pini@disi.unige.it
}

\begin{abstract}
Aspect-oriented techniques are widely used to better modularize object-oriented programs by introducing crosscutting concerns in a safe and non-invasive way, i.e., aspectoriented mechanisms better address the modularization of functionality that orthogonally crosscuts the implementation of the application.

Unfortunately, as noted by several researchers, most of the current aspect-oriented approaches are too coupled with the application code, and this fact hinders the concerns separability and consequently their re-usability since each aspect is strictly tailored on the base application. Moreover, the join points (i.e., locations affected by a crosscutting concerns) actually are defined at the operation level. It implies that the possible set of join points includes every operation (e.g., method invocations) that the system performs. Whereas, in many contexts we wish to define aspects that are expected to work at the statement level, i.e., by considering as a join point every point between two generic statements (i.e., lines of code).

In this paper, we present our approach, called Blueprint, to overcome the abovementioned limitations of the current aspect-oriented approaches. The Blueprint consists of a new aspect-oriented programming language based on modeling the join point selection mechanism at a high-level of abstraction to decouple aspects from the application code. To this regard, we adopt a high-level pattern-based join point model, where join points are described by join point blueprints, i.e., behavioral patterns describing where the join points should be found.
\end{abstract}

Keywords: Aspect-Oriented Programming, Join Point Selection Mechanisms, Join Point Models.

\section{INTRODUCTION}

Aspect-oriented programming (AOP) is a powerful technique to better modularize object-oriented programs by introducing crosscutting concerns in a safe and noninvasive way. Each AOP approach is characterized by a join point model (JPM)

Cite this article as follows: Walter Cazzola and Sonia Pini: On the Footprints of Join Points: The Blueprint Approach, in Journal of Object Technology, vol. 6, no. 7, Special Issue: AspectOriented Modeling, August 2007, pages 167-192, http://www.jot.fm/issues/issues_2007_8/article7 
consisting of the join points, a mechanism for selecting the join points (pointcuts) and a mechanism for raising effects at the join points (advice) [26]. Crosscutting concerns might be poorly modularized as aspects without an appropriate join point model that covers all the interested elements. A pointcut definition language allows the programmer to select all the desired join points.

In most of the $\mathrm{AOP}$ approaches, the pointcut definition language allows the programmer to select the join points on the basis of the program's lexical structure, such as explicit program element names. The dependency on the program syntax renders the pointcut definitions fragile [15] and strictly couples an aspect to a specific program and language, hindering its reusability and evolvability [11]. The required enhancement should consist of developing a pointcut definition language that supports join point selection on a more semantic way [1]. To provide a more expressive and semantic-oriented selection mechanism requires a language that captures the base-level program behavior and properties abstracting from the syntactic details. Several attempts (e.g., $[24,18,17,11,23,14])$ in this direction have been investigated but none of these completely solve the problem. They focus on specific behavioral aspects such as execution trace [4] and dataflow [10] neglecting some others. Moreover, they still rely on name conventions and on the knowledge of the implementation code. We think that the problem could be faced and solved by selecting the join points on an abstract representation of the program, such as its design information.

In this paper, we present a novel aspect-oriented framework, called the Blueprint, and in particular its join point selection mechanism that allows the selection of the join points abstracting from implementation details, name conventions and to some extent from the base-program structure. In particular the aspect programmer can select the join points of interest by describing their supposed location in the application through UML-like ${ }^{1}$ descriptions (basically, activity diagrams) representing computational patterns on the application behavior; these descriptions are called blueprints. The blueprints are just patterns on the application behavior, i.e., they are not derived from the system design information but express properties on them. In other words, we adopt a sort of enriched UML diagram to describe the application control flows or computational properties and to identify the join points inside these contexts. Pointcuts consist of an enumeration of join points from a set of blueprints. Thus, they are not tailored on the application syntax and structure but only on its behavior.

The rest of the paper is organized as follows: in section 2 we investigate the limitations of some of the other join point models, in section 3 we present the Blueprint framework and how it works whereas in section 4 we show the inner process of selecting the join points and the aspect weaving at the join points. In section 5 we show the framework at work; section 6 considers a few of related works.

\footnotetext{
${ }^{1}$ Please note, the Blueprint approach adopts a UML-like description since we use a subset of the UML activity diagrams with some differences in their meaning and in general they have a different role, all these differences should be clear going on in the reading.
} 
Finally, in section 7 we draw out our conclusions and discuss possible future work.

\section{LIMITATIONS OF THE JOIN POINT MODELS}

The join point model, in particular its pointcut definition language, has a critical role in the applicability of the aspect-oriented methodology. The pointcut definition language allows to determine where a concern crosscuts the code.

Pointcut definition languages have evolved to improve their expressivity, their independence of the base code and the general flexibility of the approach. The first generation of pointcut definition languages (please see [13]) were strictly coupled to the application source code because they allow the selection of join points only on the signature of the program elements (by enumeration). To reduce the coupling problem, the use of wildcards has been introduced (as in, AspectJ [12], and HyperJ [19]). This technique has slightly reduced the coupling problem but has introduced the necessity of naming conventions. Unfortunately, naming conventions raise a new problem since they are not checkable by the compilers and their adoption cannot be guaranteed. Recently, some aspect-oriented languages (e.g., AspectJ 5, AspectWerkz [27]) adopted meta-data to identify the join points. This approach decouples the aspects from the base program syntax and structure; the meta-data is used as a placeholder to mark a join point to be easily selected among the others. This technique does not solve the problem; it just shifts the coupling from the program syntax to the meta-data syntax. Moreover, this approach breaks in an explicit way the obliviousness [6] property ${ }^{2}$. To get obliviousness the aspect programmer should be unaware of the base program structure and syntax to apply the aspects, and vice versa.

The coupling problem brings forth another problem, called the fragile pointcut problem [15]. When the pointcut definition strictly depends on the base program, any change to the program will affect the pointcut capacity of grabbing the expected set of join points. The fragile pointcut problem is a serious inhibitor to evolution of aspect-oriented programs. Pointcuts are deemed fragile when seemingly innocent changes to the base program, such as renaming or relocating a method, break a pointcut such that it no longer captures the join points it is intended to capture [3, 11]. Pointcuts are similarly considered fragile when a just introduced join point should be captured by an existing pointcut but it fails to do so. This implies that all pointcuts of each aspect need to be checked and possibly revised whenever the base program evolves, since they could break because they capture a set of join points based on some structural and syntactical properties that any change to the base program can alter. This problem occurs independent of whether wild-card expressions are used.

\footnotetext{
${ }^{2}$ Please note that the value and acceptance of "obliviousness" as a key AOP property is still debated (e.g., [21]) but we consider it a desirable feature to some extent and therefore to be considered.
} 
In this situation, the aspect programmer must have a deep knowledge of the base program to be sure that his/her pointcuts work as expected. Moreover, most of the join point selection mechanisms (e.g., in AspectJ) are suitable to select join points that are at the object interface level but they badly fit the need of capturing join points expressed by computational patterns, such as inside loops or after a given sequence of statements.

Pointcut definitions heavily rely on how the software is structured at a given moment in time. In fact, the aspect developers assume the structure of the base program when they define the pointcuts; the name conventions are an example of this assumption. They implicitly impose some design rules that the base program developers have to respect when they evolve their programs to be compliant with the existing aspects and to avoid the selection of more or less join points than expected.

We believe that aspect programmers need to be, as much as possible, unaware of base code details and evolution - we call this extension to the obliviousness definition application syntactic obliviousness. To achieve application syntactic obliviousness the aspect programmer should be unaware of the base program structure and syntax to apply the aspects and vice versa. From our point of view, the base program is seen as a gray-box, where it is possible to see the high-level information about it, such as its behavior, its design information, and so on, but it should be impossible to see the base code details. In this way, if an aspect needs to be applied to a program, the lack of knowledge of its internals would prevent the use of syntactic pointcuts. Of course, total obliviousness will drive to more reusable code but will also increase the number of join points selected but not desired [21]. To address this objective, we need a total separation of pointcut definition from aspects tied to lexical properties of the source code.

From the reported considerations, it is fairly evident that the main problems of current join point models are due to the pointcut expression languages, that often do not offer the right degree of abstraction with respect to the base program. Hence, we think that the next step of aspect-oriented methodology consists of extending the pointcut definition language to support join point selection on the basis of a semantic query. To solve all the previously cited problems (that is, coupling problem, fragile pointcut problem and obliviousness reducing), we propose a novel approach to the join point selection, called Blueprint, based on describing a portion of the base program behavior, through a model, where to identify the join points of interest.

As stated in [1], working at the model level offers promising possibilities to start abstracting away from the concrete syntax. This means that the model information (such as, UML diagrams) describing a program is independent of its implementation, both of the programming languages characteristics and of the names used at the code level. The model-based pointcut definitions are both less fragile and less coupled, because they are not defined in terms of syntactic description of base program characteristics. Moreover, model-based approaches promote the application of the syntactic obliviousness property because they do not need to know the code details. 


\section{THE BLUEPRINT FRAMEWORK}

In the Blueprint approach, we select the join points by providing a template (a blueprint) of the base program behavior/computational models which describes where the join points could be found. This approach does not only allow precise location of where we are looking for but also of describing some context information, such as, what happens before or after the join point: a certain point in the computation is a join point if and only if all the specified context conditions are verified. The Blueprint framework is based on our previous work [2] and it is completely detailed in [20]. This section gives an overview of the Blueprint aspect-oriented language and describes how to use it.

\section{The Blueprint Aspect-Oriented Approach.}

The Blueprint aspect-oriented language permits the selection of the join points of interest by describing their supposed location in the application through a UML activity diagram representing patterns on the application behavior, called join point blueprint. These join point blueprints are not subsets of the application design information. They do not describe the application behavior, rather they describe the desired properties and behaviors we are looking in the application.

The Blueprint framework foresees a matching and unification phase that permits to perform queries such as "print the value of a variable used in a loop test condition and modified in the loop body". This kind of query is expressed describing the context we would like to get and the position where we would like to raise effects. To carry out this kind of query we have to compare our description with the source code of the base-program during the weaving process. The Blueprint language can be used on the bytecode as well since it can be univocally decompiled (modulo semantic equivalence) by appropriate tools, e.g., by Jode ${ }^{3}$.

In our approach, we do not need to use position qualifiers such as before and after advice to indicate where to insert the concern inside the base code. Because we describe the context, we can either locate the join points exactly where we want to insert the new code or, to highlight the portion of behavior we want to replace.

\section{Blueprint Join Point Model}

The Blueprint framework recalls the AspectJ terminology but some terms are used with a slightly different meaning. Introductions and advice keep their usual meaning whereas join points and pointcuts have slight deviations. The Blueprint join points are hooks where the code may be added rather than well-defined points in the execution of a program where effects can be raised. In AspectJ, the considered join

\footnotetext{
${ }^{3}$ Jode is available at http://jode. sourceforge. net.
} 


\begin{tabular}{|l|}
\hline Blueprint Description Language: Terminology and Elements Description \\
\hline join points \\
hooks where the crosscutting concerns will tangle the application. \\
\hline join point blueprints (in short blueprints) \\
patterns describing a set of join points in terms of their application context. \\
They provide an incomplete and parametric representation of an application \\
behavior portion. \\
blueprint space \\
it is the set of all join point blueprints defined on a given application. \\
pointcuts \\
crosscutting concerns to apply at the join points when the associated pointcut \\
is true. \\
advice blueprint space selecting a set of join points. \\
introductions
\end{tabular}

Table 1: Blueprint Terminology.

points are things like method and constructor calls, method and constructor executions and field references. That is, they are at the operation interface but a join point could occur everywhere in the code not only at the operation interface - the Blueprint exploits this concept. This view grants a statement-level granularity to the Blueprint join point model. In particular, we consider two different kinds of join points: the local join points that represent points in the application behavior where to insert the code of the concern, and region join points that represent portions of the application behavior that must be replaced by the code of the concern.

To complete the picture of the situation, we have introduced some new concepts: join point blueprint and blueprint space. The former is a template (a blueprint) on the application behavior identifying the join points in their context; these blueprints describe where the local and region join points should be located in the application behavior. The blueprint does not completely describe the computational flow but only the portions relevant to select the join points. The latter is the set of all join point blueprints defined on the same application. The pointcuts are a query on the blueprint space, i.e., they select some join points imported from one or more blueprints. Table 1 summarizes the concepts characterizing the Blueprint model.

The Blueprint is based on the idea that the description of the application behavior cannot be strictly coupled to the application syntactic details. It permits a loose approach to the description of the application behavior. This means that the aspect programmer can use different levels of detail during the description of a single join point blueprint by using any possible combinations of loose and tight elements. This approach permits the description of a well identified behavior tightly coupled to the application code by specifying the names of the involved elements, and a less known behavior by using meta-information to abstract from the real application code.

The join point blueprints are the key elements of the whole approach. They 


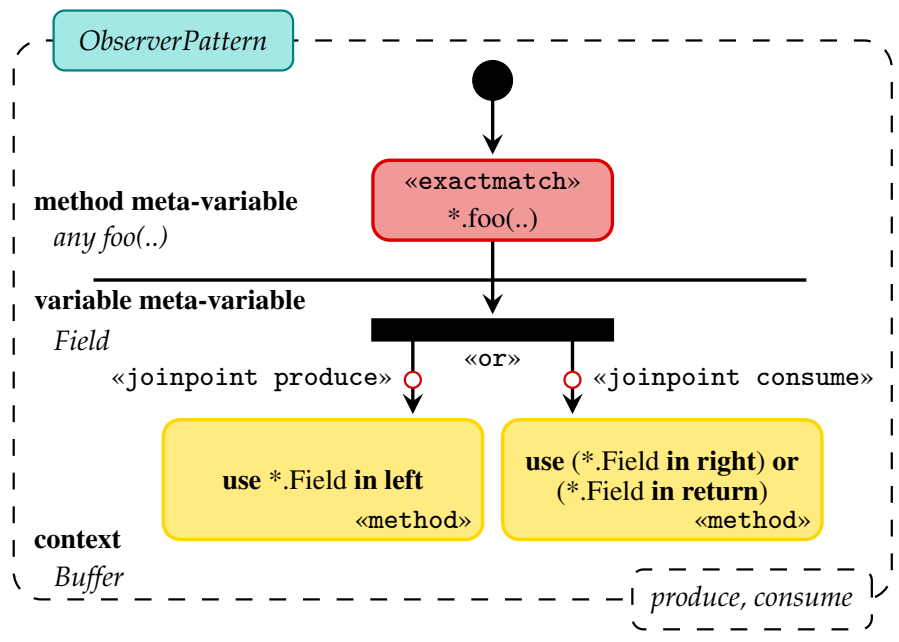

Figure 1: Sample Join Point Blueprint.

graphically depict where a join point, both local and region, should be located in the application behavior. They look like an activity diagram and to some extent they behave similarly. Conceptually, both represent part of the computational flow of the application. What differs is their use: the activity diagrams are used to model the application behavior whereas the join point blueprint matches the supposed application behavior. For this reason, some elements composing the diagram have additional meanings and the diagram itself is accompanied by context information.

A join point blueprint depicts where a join point should be located in the application behavior. Each blueprint is a diagram framed by a dashed rectangle. The diagram contextualizes the join point location by describing some crucial events that should occur close to the join point. These events will be used to recognize the join point. The frame gives some ancillary information, such as the blueprint name (at the top left corner), the join point names exposed by the blueprint (at the bottom right corner) and some meta-info (see later in the section) used by the weaver to parametrize the context and to get values from the join point. The listed join points are only exposed to the pointcut specification. The join point location is denoted by the «joinpoint name» stereotype (or by the pair «startjoinpoint name» and «endjoinpoint name» for the region join points).

Figure 1 shows a very simple join point blueprint that does not fully illustrate the whole expressivity allowed by the formalism. For a detailed and exhaustive description, please refer to [20] chapter 4 . The following provides a brief overview of all elements that can appear in a join point blueprint.

Computational Flow Description Elements. In the join point blueprint it is possible to use a set of programming abstractions for modeling the control flow of the described behavior: conditional construct (if), cycles and loops (for, while, and so on) and object flow (swimlane). These flow abstractions are represented by the standard UML decision elements and are used to describe the application 
computational flow we desire. The aspect programmer can fill these flow abstractions with details such as the checked condition; the more details are used the easier the searched behavior can be uniquely identified. Particularly relevant is the use of the swimlanes, which permit the organization of the searched behavior in terms of the actions that should be performed by any single actor. This better contextualizes where to look for a specific part of the blueprint. Figure 1 has a swimlane that separates the call of a method from the description of what happens inside the method itself.

Loose and Tight Elements. To abstract from the application syntactic details, the Blueprint language allows to define the blueprints in an incomplete/abstract way, i.e., it is possible to describe the computational flow by using a loose approach. Analogously, to get more in touch with the searched behavior it is possible to specify all the necessary details to skim among similar portions of code, i.e., it is possible to use tight elements in the blueprint description.

The Blueprint allows the aspect programmer to use meta-information to depict a blueprint of the searched behavior without referring to the application element names and types. This independence has been achieved through template actions and loose transitions. A loose transition is a line with a stick arrowhead connecting two action states in the blueprint; it indicates that the target action state follows the source action state but not immediately, i.e., zero or more not relevant (to the join point localization) instructions could occur before the target action state, the number of instructions that could occur is limited by the (optional) transition scope. A template action is a yellow action state containing a template-statement and optionally a scope indication. The template-statement is a statement defined by the following regular expression:

\section{use $\left((A\right.$ in $\left.B)[\text { or } \mid \text { and }(A \text { in } B)]^{*}\right)$}

where A can be either a name taken from the application code or a meta-variable (see later in the meta-information section); B represents where the name A should be found in the application code and can be (with the obvious meanings): booleancondition, left, right, index, return, or statement.

All the blueprint loose elements have a scope denoted by a stereotype, which limits the searching area of the blueprint portion inside the application code. There are two kinds of scope: «method» and «block». The former limits the searching area to the extent of the current method body (default behavior) whereas the latter to the extent of the current code block.

An aspect programmer can use a template action or a loose transition when he is not interested in a well-defined computational pattern but only on few characteristics, like the check for a condition or the use of a specific variable in a return statement. Otherwise, he can specify all necessary details to skim among similar portions of code through the use of actions and tight transitions.

An action is a red action state containing one or more Java statements which 
must exactly match a sequence of instructions in the application code. To better distinguish actions from template actions, the action is always decorated (at the top) by the «exactmatch» stereotype. The statements inside the action can use both meta-variables and real names. A tight transition is a line with a solid arrowhead connecting two action states of the blueprint; it indicates that the target action state follows immediately the source action state inside the application control flow.

Note that loose and tight elements can be mixed inside a blueprint. To avoid ambiguities the tight elements have higher priority than the loose elements, the «block» has higher priority than the «method»scope qualifier.

Figure 1 shows three action states: an action and two template-actions; the action describes a call to a method whose name is unknown (i.e., foo is a metavariable). All the transitions are loose (they all have a stick arrowhead) so we are just looking for a loose pattern with a given and incomplete sequence of statements.

Flow Operators. Complementary and alternative behaviors can be described by using the flow operators provided by the language: and and or. These operators resemble the UML fork element decorated, respectively, by the «and» and «or» stereotype. The and operator allows the programmer to describe multiple behaviors that will be searched into the application computational flow; whereas the or operator can be used to describe alternative behaviors. In the first case we have to match all branches to consider the operator matched. In the second case, at least one match is needed. The flow operators are useful to separately describe concerns that can be tangled inside the application code. Figure 1 shows an or operator with two branches.

Join Points. The Blueprint language allows the definition of a join point every point between two instructions. Two kinds of join points are considered: local and region join point. A local join point is represented by an empty circle on transition labeled by the «joinpoint jp_name» stereotype; it specifies the exact point where the advice code will be inserted when the blueprint matches the application computational flow. A region join point represents a portion (region) of the application behavior that will be replaced by the advice code when the blueprint matches the application computational flow. Two stereotypes «startjoinpoint jp_name» and «endjoinpoint jp_name» denote the borders of the region.

The location of the join point is not ambiguous when the join point stereotype is attached to a tight transition since it strictly coupled the source and target action states: the latter follows immediately the former. Therefore the join point is exactly between the last matched statement of the source action state and the first matched statement of the target action state. The situation is more complex when the join point stereotype is attached to a loose transition since we do not assume anything about where the next statement will be with respect to the join point location. To better describe the join point location the stereotypes can be combined with the location modifiers: «source» and «target» to express the join point vicinity relation. 
Figure 1 shows a couple of local join points: produce and consume. They are both on a loose transition but exploit the default qualifiers: «method» and «target».

Meta-Information. Inside the frame of the join point blueprint there is a set of meta-information associated to each swimlane useful to decouple the blueprint from the application code and to contextualize where to look in the application code for the blueprint.

The meta-information allows the programmer to describe the blueprint decoupled from the application code. The weaving mechanism will provide an unification between the meta-variable names and the variable names used in the application code, if it will be possible.

In each swimlane, there are up to five sections containing meta-information: context, variable meta-variable, method meta-variable, type meta-variable and type binding. The context section contains the name(s) of the class(es) where to look for the computational flow described in the swimlane. The meta-variable sections contain a pool of variable names that can be used in the blueprint instead of the names coming from the code; these are called meta-variables since they contain names and not values. The meta-variables can refer to variable and method names or to types. In the type binding section, it is possible to express bindings among the variable and method meta-variables and the type meta-variables. This mechanism permits to realize polymorphisms on the variable and method meta-variables, e.g., given a type meta-variable named foo associated to int and String we could declare a variable meta-variable bar of type foo that will match both strings and integers variables in the code. The language assumes that all names present in the blueprint but not present in the meta-information sections are real application element names.

The blueprint in Figure 1 has two swimlanes. At the top, there is a method meta-variable (foo) - please, note that the any return type denotes any possible return type. At the bottom, there is the Field variable meta-variable and a context specification Buffer that confines the match for this swimlane to this class.

\section{Blueprint Aspects}

An aspect is the modular unit that crosscuts other modular units. Similar to AspectJ, the aspect declaration looks like a class declaration.

Listing 1 covers the basics of what an aspect can contain. An aspect consists of method and field declarations (rows 2-3), a join point blueprint import section (rows 4-7), pointcut definitions (rows 9-11), the advice (rows 13-15), and finally the code for the introductions (rows 16-18).

The method and field declaration section contains methods and fields local to the aspect. These can be used in the advice section but these methods cannot access the base-program private elements since they are in the aspect scope. 


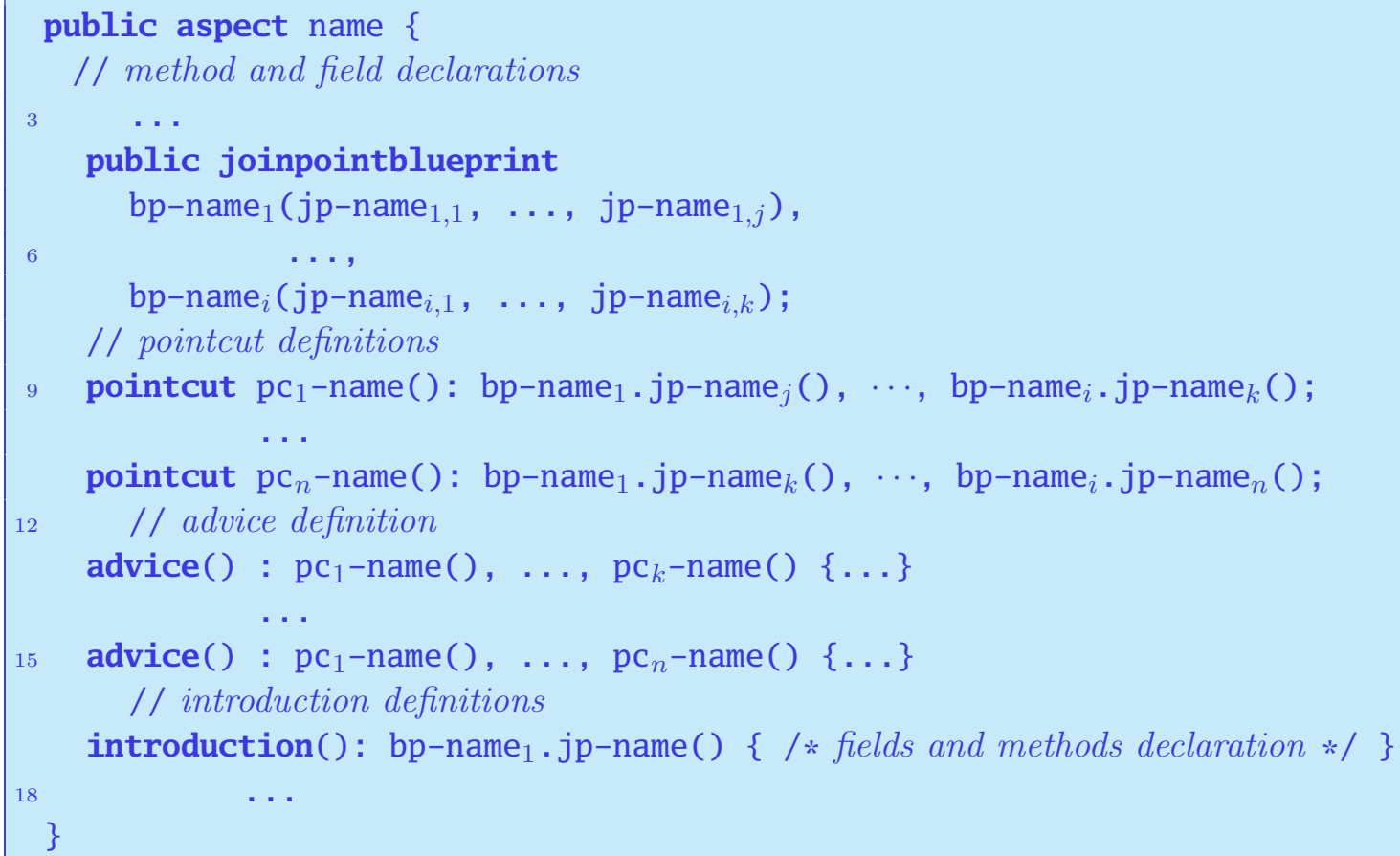

Listing 1: An Example of Aspect Definition by using the Blueprint Language.

In the blueprint import section, introduced by the keyword joinpointblueprint, we list the join points out of the blueprints that will be used inside the aspect. To list a blueprint name without parameters is to import all of its join points, otherwise only the listed ones are imported.

In the pointcut section, we associate a pointcut, i.e., a query on the blueprint space, to the join points imported in the specific section. The association is basically achieved by listing the desired join point names.

The advice section defines crosscutting behaviors that should be introduced at the selected join points. An advice is associated to a pointcut or to a list of join point names imported from a blueprint. The code of the advice runs at every join point picked out by its pointcut.

Finally, the introductions, introduced by the introduction keyword, allow the definition of new methods and attributes that will be added to the application code during the weaving process. This ancillary code can assess the private information of the application code where they are woven.

The Blueprint provides a simple reflective API (reported in Table 2) to access context information like the join point signature ${ }^{4}$ and the meta-information specified in the blueprint, such as variable and method meta-variables, through a special reference: thisJoinPoint(jp-name). thisJoinPoint (...) can be used both in-

\footnotetext{
${ }^{4}$ Please note that in our case we are not concerned about the method captured by the pointcut, but rather about the method that contains the captured join point.
} 


\begin{tabular}{|l|}
\hline Blueprint reflective API: Description \\
\hline $\begin{array}{l}\text { public Signature getSignature() } \\
\text { returns the signature of the method that contains the matched join point. }\end{array}$ \\
\hline $\begin{array}{l}\text { public Method getMethod() } \\
\text { point. }\end{array}$ \\
\hline $\begin{array}{l}\text { public SourceLocation getSourceLocation() } \\
\text { returns the source location corresponding to the join point. } \\
\text { returns a String representing the kind of join point (e.g., local or region). } \\
\text { public String getKind() } \\
\text { returns the source code corresponding to the region join point, null otherwise. } \\
\text { returns the signature of the method meta-variable mtd. } \\
\text { public Signature getUnifiedSignature(String mtd) } \\
\text { Returns a Method object representing the mtd method meta-variable. } \\
\text { Returns a Type object that represents the declared type for the application } \\
\text { variable unified to the varname variable meta-variable. }\end{array}$ \\
\hline $\begin{array}{l}\text { public Type getUnifiedType(String varname) } \\
\text { Rethod getUnifiedMethod(String mtd) }\end{array}$ \\
\hline
\end{tabular}

Table 2: Blueprint Reflective API: thisJoinPoint(jp) Methods

side advice and introductions. In the advice the parameter represents one of the join points specified in the advice, whereas in the introduction it can refer to every join point declared inside the aspect. The weaving process will create a connection between the meta-variables and the matched application elements. Therefore, by using the meta-variables (through the reflective API) corresponds to using the real elements. At the moment, the available reflective API is still quite limited but some extensions are under development.

\section{BLUEPRINT MATCHING AND WEAVING}

One crucial component in AOP is the weaver. Given a set of target programs and a set of aspects, the weaver introduces the code of the advice at the captured join points in the target programs during the weaving process.

Even if the join point blueprints are language independent, the weaving process strictly depends on the program it has to modify. At the moment, we have chosen the Java programming language but in the future we are planning to extend the approach to many other languages. The Blueprint weaving process consists of the following phases:

- pre-weaving phase: the abstraction level of the join point blueprint and of the Java bytecode is equalized;

- matching phase: the matching is performed by traversing the model/graph of 
the blueprint and the model/graph of the program in parallel;

- advice weaving phase: the advice code is inserted at the captured join points.

Pre-Weaving Phase. The base program and the join point blueprints are at different levels of abstraction. To fill this gap and allow the weaving, it is necessary to build a common representation for the base program and the join point blueprints. The abstract syntax tree (AST) perfectly fits the problem; both source code (through its control flow graph) and join point blueprints can be represented by AST-like descriptions.

The AST of the base program is simply developed by using a parser and AST generator. To generate the AST for the blueprints is more complex. Any UML diagram can be represented as a graph because it is defined by the UML metamodel which is a graph where the nodes are meta-classes and the edges are metarelationships, but it is not possible to generate such kind of a tree from the join point blueprint because of the loose elements. To overcome this problem, we have created a graph where each transition becomes a labeled graph edge, each action becomes a graph node containing the AST nodes generated by the Java instructions contained in the action. This kind of graph node is called a complex node; each template action becomes a simple node that does not contain AST nodes but some information about the scope of and the kind of statement looked for by the corresponding template action. It is necessary to differentiate the graph nodes because we cannot define an AST to describe a template action, because it does not contain real Java instructions. Labels represent the scope information, join point location and so on. We call this representation: Blueprint_Graph.

Matching Phase. After obtaining the same level of abstraction for source code and blueprints, the next step is to find all matchings among each Blueprint_Graphs, generated in the previous phase and the application AST.

Our matching algorithm is not a simple algorithm to match two graphs, because we do not have the same kind of graphs but a graph and an AST representation. To solve this, we developed a special matching algorithm, called multiple spread tree inclusion, that looks for:

- a tree matching between portions of the application AST and the complex nodes of the Blueprint_graph; and

- a matching between the Blueprint_graph simple nodes and portions of the application AST.

These matchings are driven by the edges connecting two Blueprint_Graph elements in a depth-first visit and the search area defined by the context meta-information.

During the matching algorithm we have to associate the meta-variable names used inside the blueprints to the real names used inside the application (unification 


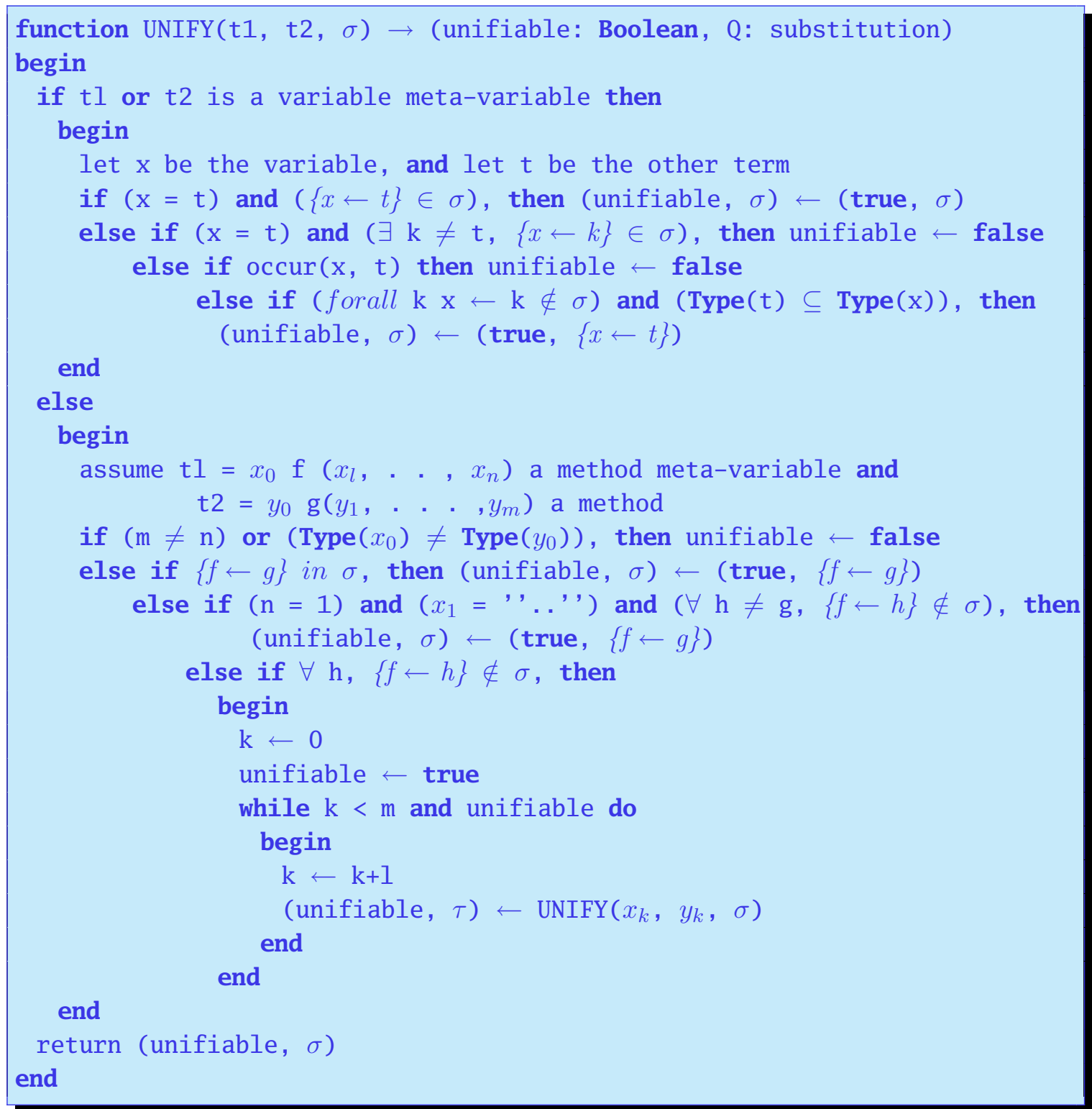

Listing 2: The Blueprint Unification Algorithm

process). The unification algorithm used in the Blueprint framework (listing 2 shows its pseudocode) is based on the well-known Robinson's unification algorithm [22].

Beyond using the unification, our matching algorithm is strongly based on backtracking, since, to find all the possible matchings it must try all the tree branches. Every blueprint matching is called possible matching as long as the last action state of the blueprint has not been matched, from this point the matching is a sure matching.

Turning back to the single steps of our matching algorithm, in the first point we search a real matching between the AST nodes of a complex node and, a portion of application AST nodes, obviously, but the unification operations. This first step 
is a tree pattern matching problem [8]. We use an algorithm based on tree pattern matching presented in [16].

Advice Weaving Phase. This is the last step of the weaving phase. During this step the advice code is inserted into the application. This final step starts only when the previous step obtains a sure matching for the considered blueprint. To develop this step, the framework uses all information about join points and unifications stored during the matching phase and the advice source code.

- The meta-information are used to identify the method affected by the advice;

- the unifications are used to unify the meta-variable names used inside the advice code, to the names used inside the application source code; in this way the code of the advice will refer to the code elements; and

- the advice source code, after the unification, is inserted into the local copy of the right file, in the right position, corresponding to the considered join point.

To maintain the application source code unchanged, the Blueprint framework uses a local copy of every application source file to insert the advice code into the join points. After the introduction, the modified files are compiled on-the-fly by using the javac compiler. The .class files are superseded to the original application .class files. This last step exploits a Java library, called RECODER ${ }^{5}$ to modify and parse the original application files. This library is already used by other aspect-oriented tools, such as EAOP [5].

A complete description of the Blueprint weaving can be read in [20] chapter 5 .

\section{BLUEPRINT AT WORK}

To stress the Blueprint potential in this section we present three examples. The first one is a classical tracing aspect that demonstrates how the Blueprint can carry out all of the modularization capabilities offered by other aspect-oriented approaches. The second example, debugging and monitoring the execution of a program, should show the capability of the blueprints for capturing join points that the other approaches cannot deal with. The last example shows the Blueprint reflective API at work.

\section{The Blueprint Tracing Aspect.}

Usually during the development, programmers insert debugging messages in their code (e.g., to notify the beginning and the end of the method or constructor execution) to better follow and test the execution of the application.

\footnotetext{
${ }^{5}$ Available at http://recoder. sourceforge . net.
} 


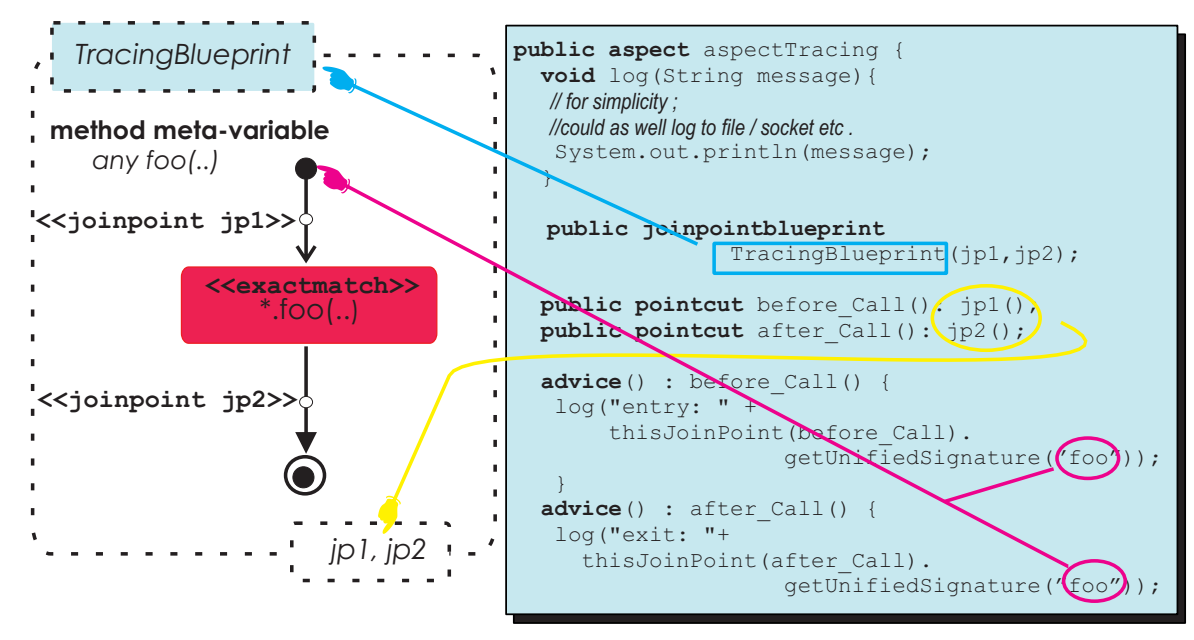

Figure 2: A Simple Tracing Blueprint and Aspect.

Even though this is a simple debugging mechanism, it has a number of drawbacks:

- the implementation of this feature is scattered over many classes of the system,

- many classes are cluttered with the message generation code, and

- after development it is difficult to safely remove the debugging code.

In short, the implementation of this conceptually simple feature leads to severely tangled code. To this regard, we developed a simple tracing aspect consisting of a join point blueprint definition and an advice.

The TracingBlueprint, showed in Figure 2, describes all method calls irrespective of their name, signature, target object and where they are called and defined. The method meta-variable foo during the weaving process is unified to the called method signature, and inside the advice code it is possible refer to it and, by applying the getSignature() method, visualizing the full name of the application method. The blueprint defines two join points: jp1 and jp2; at these join points the computational flow will be traced (woven advice).

\section{Debugging and Monitoring the Knapsack Algorithm Execution.}

The typical debugger's functionality, such as variable and state watching, and tracing can be easily realized through a Blueprint aspect.

The right half of Figure 3 shows part of Rolfe's solution ${ }^{6}$ to the well known Knapsack problem. The Knapsack problem, as well as any other problem in combinatorial optimization, offers several temporary values to be monitored during the execution,

\footnotetext{
${ }^{6}$ Available at http://penguin. ewu . edu/ trolfe/Knapsack01/index.html.
} 


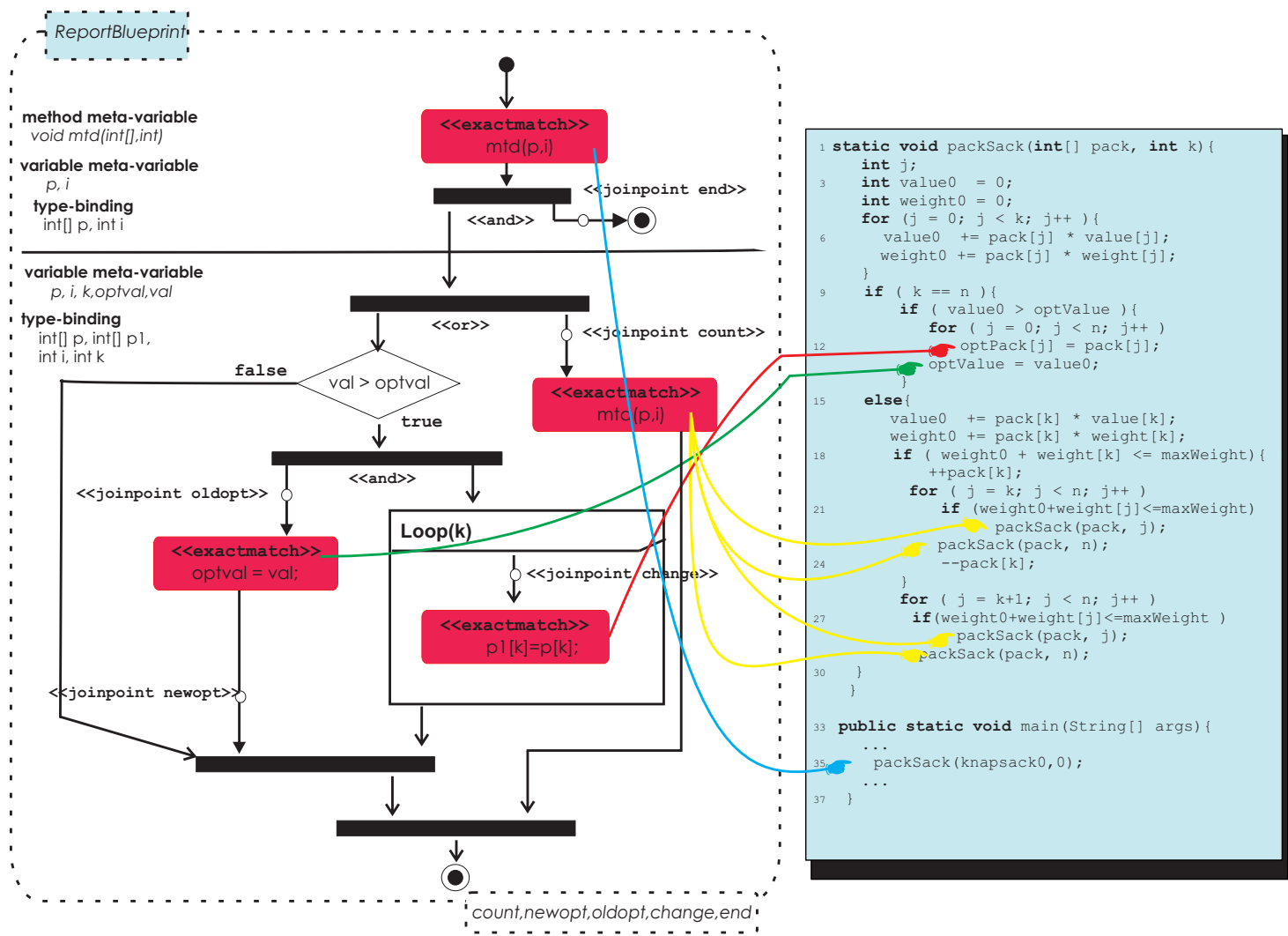

Figure 3: The Report Blueprint and the Matched Code.

e.g., the evolution of the optimal solution, the changes to the optimal value and the number of iterations to get the optimum. It is fairly evident that these issues can be treated as a problem of breakpoint settings and variable watching. In particular, a change in the current optimal solution depends on finding a better approximation to the optimum as checked at row 10. This represents a relevant context information exploited by the blueprint in the left part of Figure 3 to contextualize the event independently of the names used in the code; note that val and optval are variable meta-variables that, during the unification phase, will match value 0 and optValue in the code, respectively. The Blueprint aspect, shown in Listing 3, will exploit, through reflection, the value matched by optval at the oldopt() and newopt() join point to print the optimum before and after the change. The changes to the current optimal solution are detected and reported in a similar way. To calculate the number of iterations to get the optimum we have to look for the recursive invocations of the main method (packSack()), to count them and to report the total at the end. The count () and end() join points are, respectively, where to increment the total number of iterations and where to print it. The aspect will introduce a new variable (counter) to the application and the advice will work on this variable, incrementing it at the count () join point and printing it at the end() join point. 


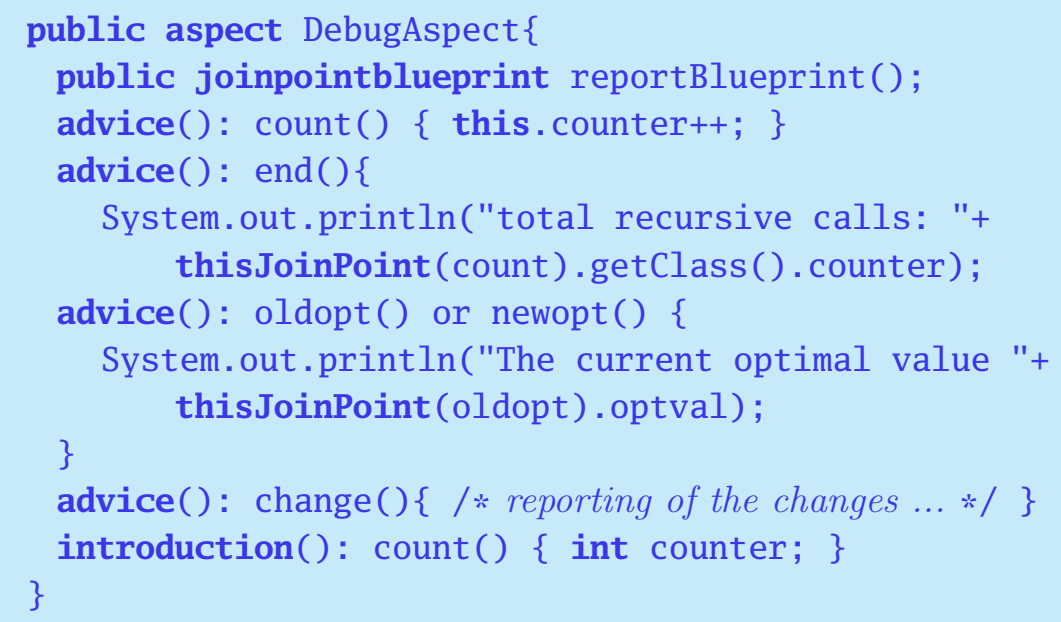

Listing 3: The DebugAspect Used to Monitor the Knapsack Algorithm.

Parallelizing the Mandelbrot Algorithm.

Another interesting example application of the Blueprint approach is the parallelization of a method. Let's suppose one is interested in parallel execution of a method. By using the Blueprint region join point we can indicate which portion of the method behavior can be executed concurrently and in another thread/process.

In [9], Isberg adopts AspectJ 5 to develop a similar idea; in particular, he uses the annotations to mark the method (and not part of its body) to render parallel. The Blueprint approach is more flexible that AspectJ because any portion of a method can be parallelized, not only the whole method invocation.

We consider the classic example of paralleling the rendering of the Mandelbrot fractals. Our scope is to draw the fractal by using four threads, one for each fourth

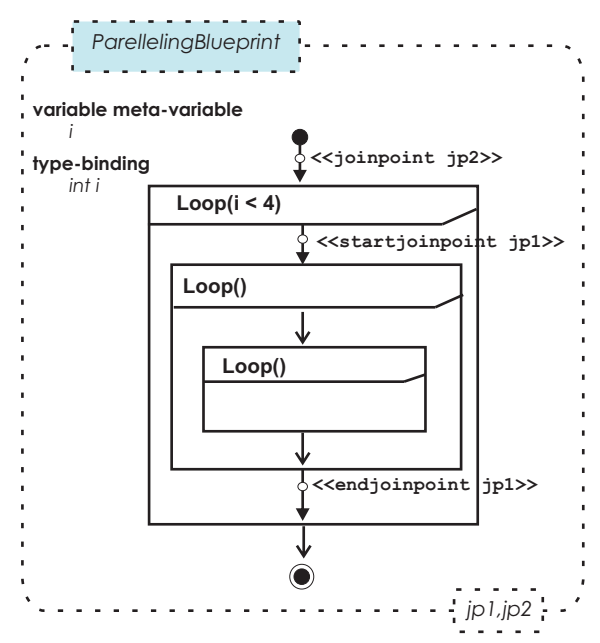

Figure 4: The Blueprint to Paralleling a Method 


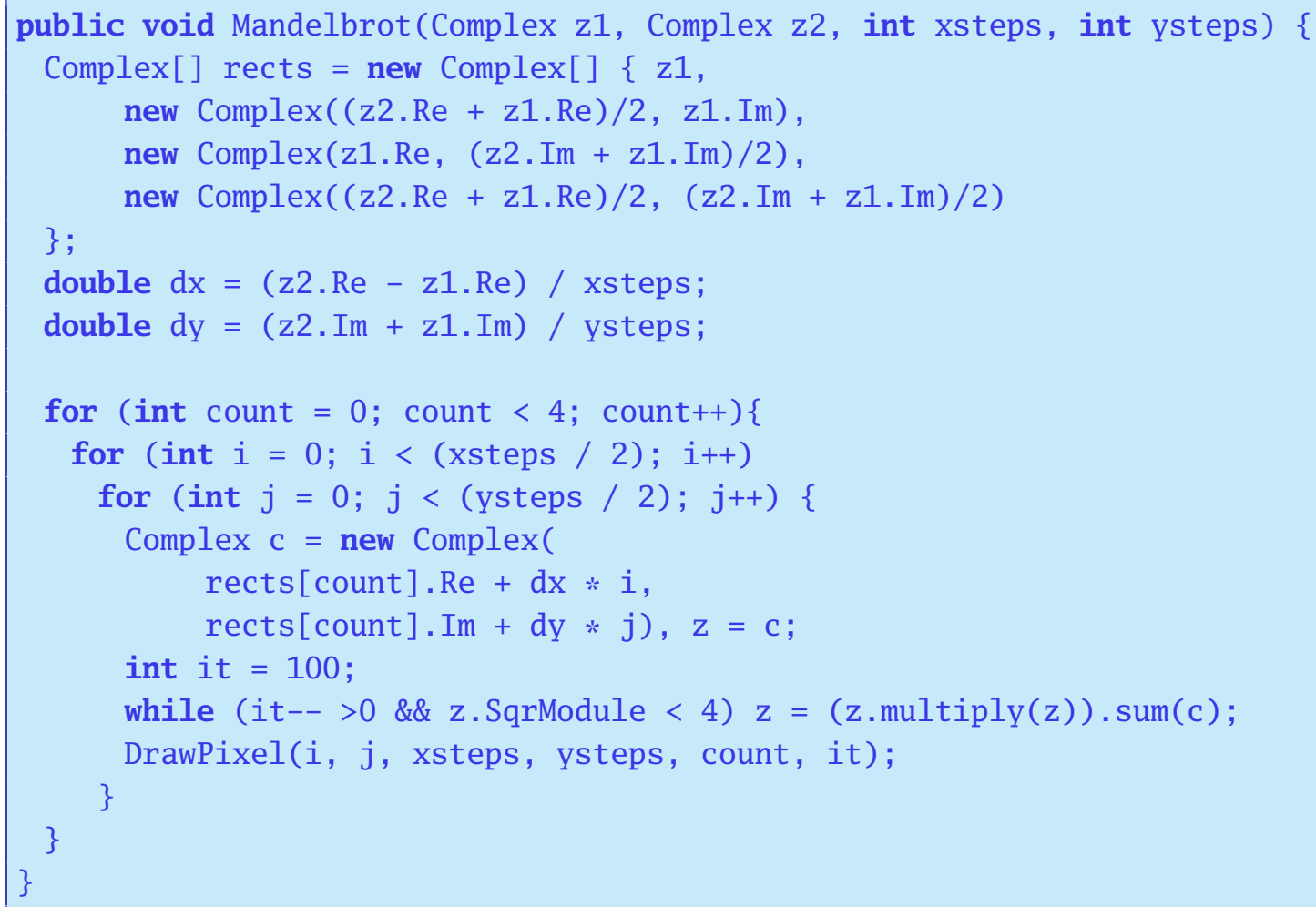

Listing 4: The Method to Calculate a Mandelbrot Fractal.

of the drawing area. The Mandelbrot () method, reported in listing 4, computes the fractal given a region in the complex plane (bound by the two complex numbers $\mathrm{z} 1$ and $\mathrm{z} 2$ ). The implemented algorithm subdivides the complex plane in four regions (whose upper left corners are contained in the rects array), and performs the classic Mandelbrot algorithm on each of them.

The parallelization is based on the presence of two new classes: ParallelTask and ParallelInfrastructure that we add to the system. These classes create the necessary parallel infrastructure; the former represents a skeleton for the thread execution with an empty run() method, that will be filled with the code extruded by the aspect (listing 5, rows 21-23), the latter deals with a ThreadPool of four threads, as required. These two classes are not relevant to the discussion and for sake of brevity they are not reported, the details can be found in [20] chapter 6 .

We use the blueprint and the aspect showed in Figure 4 to identify the portion of the method and to execute it as an asynchronous method. To do this, we must locate two join points:

- a local join point (called jp2) before the first statement will be added the creation of a new instance of the parallelinfrastructure class, and

- a region join point (called jp1) that enclose the code portion that will be replaced by the code to start a thread with the extruded code as the body of 


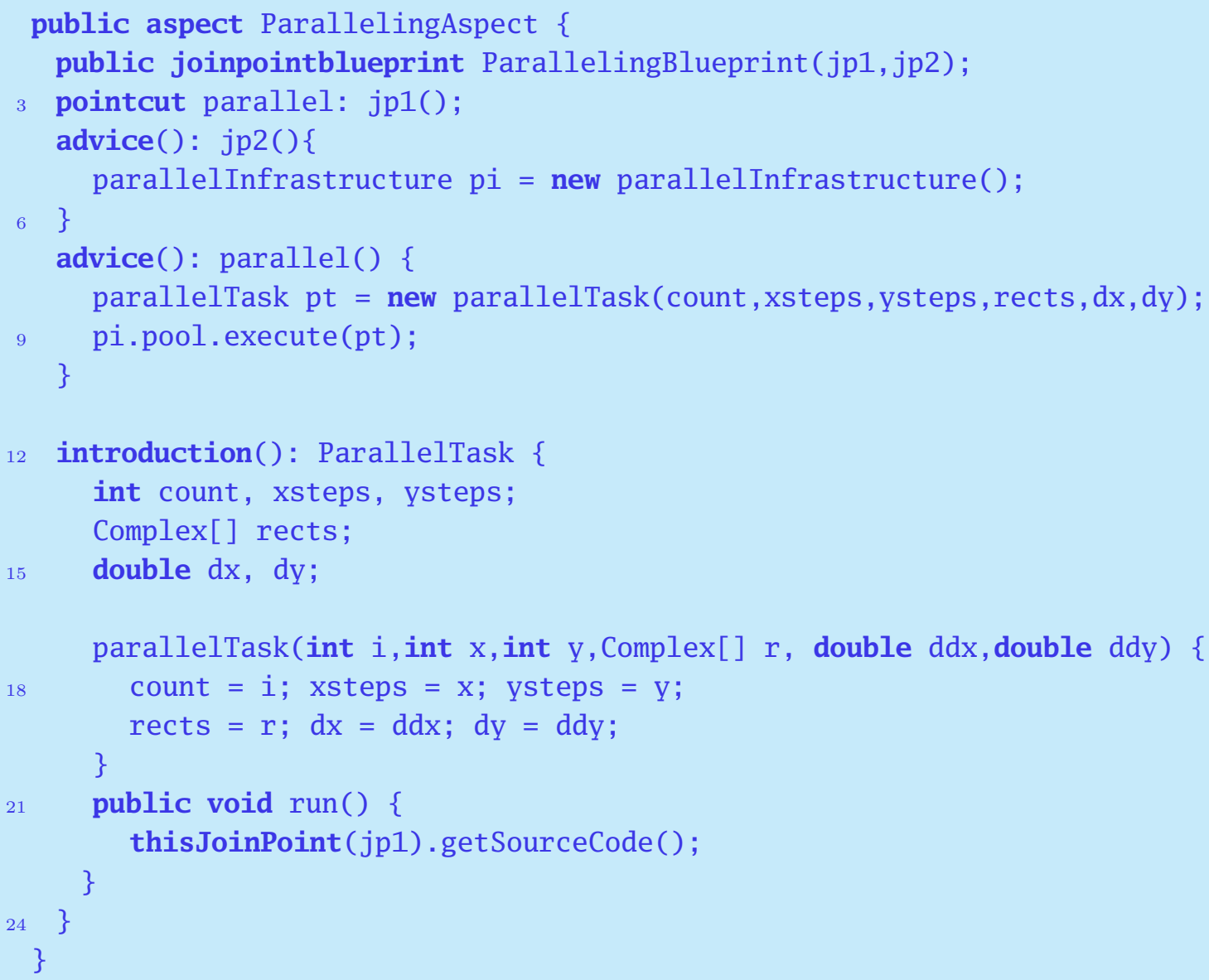

Listing 5: The Paralleling Aspect.

the run() method (see Listing 5).

The introduction of the ParallelingAspect aspect (see Listing 5 rows 12-23) acts on the ParallelTask class by:

- adding six new fields (rows 13-15),

- adding a new constructor method (rows 17-20), and

- substituting the empty run() method with the run() method with the reflectively extruded code (rows 21-23); note that introducing a method already present in the class produce a local overriding of sorting.

\section{RELATED WORK}

The Blueprint framework is not the first attempt of dealing with the limitations of the current join point selection mechanisms. In this section we report some of the most significant attempts, without pretending to be exhaustive. 
In [18], Nagy et al. propose a new approach to AOP by referring to program units through their design intentions to answer the need of expressing semantic pointcuts. Design intention is represented by annotated design information, which describes for example the behavior of a program element or its intended meaning. Instead of referring directly to the program, their approach provides a new language abstraction to specify pointcuts based on some design information. Design information are inserted inside the base program using annotations and they are associated manually, derived on the presence of other design information and, through superimposition. The key benefit of this approach is that it reduces direct dependencies between the crosscutting concerns and the program source. Unfortunately, this approach breaks the obliviousness [6] property. This property is broken because certain design information has to be specified by the software engineer, and moreover the software engineer must use a consistent and coherent set of design information for each subdomain of an application.

In [5], Douence and Südholt propose an AO approach, called EAOP, based on the observation of dynamic events. In EAOP, aspects are expressed by events emitted during execution of the base program and are defined by two languages: a crosscut language, that allows the definition of execution points where an aspect may modify the base program, and the action language, which enables the execution of the base program to be modified. The implemented tool supports four kinds of events: method and constructor calls and their return events. This approach needs a prephase to instrument the source code of the base program to generate events. We think that new kinds of event would be necessary, since these kinds are not much expressive. Moreover, the pointcut definition is strictly coupled with the base code, since it contains method and constructor names. Finally, the base program must be modified to insert the necessary events. In [4], the authors extended their work to take into consideration the whole history of the program executions. These kind of aspects are more expressive than those based on atomic points because relations between execution events can be expressed. Join points may denote not only syntactic information (e.g., instructions) but also semantic information (e.g., dynamic values). Nevertheless, the crosscut definition is also strictly coupled to the base code, since it contains, like in their previous work, program element names, such as method names.

Tourwé et al. [25] have proposed an advanced pointcut managing environment, based on machine learning techniques. They try to deal with the well-know problems of the AOP languages by including the notion of inductively generated pointcuts in the language itself. In this way developers can specify pointcuts by using a graphical interface, that offers a view on the source code, and an inductive logic programming algorithm that is responsible for computing the pointcut definition. This approach is more expressive and permits to overcome the previous problems, but it still does not permit to identify a pointcut inside the method bodies. On the contrary, since the inductive logic programming algorithm computes the pointcut definition automatically, the developer no longer has precise control over this. 
Gybels et al. [7] have dealt with the so called arranged pattern problem. Crosscutting languages use pattern matching to capture join points. This is a good technique to describe the intended semantics of a crosscut but it is still dependent of the naming convention. Gybles et al. have proposed a more flexible linguistic mechanism to implement crosscutting as patterns and consequently avoiding the exposed pattern matching problem. Essentially, their crosscut language is a logic programming language, based on Prolog. Their join point model is based on the AspectJ one, since the join points are related to key events in the execution of an object-oriented program. They use SmalTalk as a base language, and use the following join points: message receptions by an object, message sends by an object, the accessing and updating of an object's state and the execution of code blocks.

In [24], Stein et al. presented a new graphical approach to model pointcuts. Their approach deals with modeling and graphical visualization of places and conditions of crosscutting. At the implementation level, join points represent "hooks where enhancements may be added", on modeling level, join points are rendered by model elements. In particular, their approach uses UML classifiers to represent join points in structural models, and UML messages to represent join points in behavioral models. For the designation of join points they introduce a new graphical mechanism called Join Point Designation Diagram (JPDD). A JPDD contains, when fully specified, a description of structural and behavioral constraints. The structural part is described with a notation that combines the syntax of class diagrams and object diagrams, and the behavioral part is described by a notation based on sequence diagrams. The approach is loosely coupled with the base program, and follows a graphical approach like us, but by using JPDD it is not possible to identify join points inside method body, between two instructions, since they use sequence diagrams it is only possible to identifies join points on method calls.

In [14], Klein et al. presented a new semantics-based aspect weaving algorithm for hierarchical message sequence charts (HMSCs). They chose HMCS as the scenario model. Scenario languages are mainly used to describe behaviors of distributed systems at an abstract level or to capture requirements in early development stages. In this work, they used message sequence charts (MSC), that are very similar to UML 2.0 sequence diagrams, so the approach used in this paper could also be applied to sequence diagrams as well. Behaviors and aspects are defined by using MSC. An aspect defines a part of behavior that should be replaced by another one every time it appears in the semantics of the base specification. This approach suffers from several limitations: the matching process can only be performed if each join point appears inside a bounded fragment of a behavior, another limitation is that the MSC should not exhibit two non-disjoint cycles where the pointcut matches. Finally, since it is based on sequence diagram it only possible to describe message between objects.

In [17], Mohd Ali and Rashid present a general state-based join point model. The aim of their work is to expose high-level join points in the code, based on the states and state transitions of the system, by providing a state-based AOP language platform that allows such join points to be exposed. This approach turns to safety- 
critical systems, where to capture system states is an important part of the system. Since a state-based pointcut construct permits to specify criteria for join points that refer to the program's current state (i.e., run-time values). In their notion, a crosscutting system state is defined as an abstract state machine, and they use the transitions of this abstract state machine that are controlled by state guards, to identify the join points during the execution. This approach utilizes a join point model conceptually different from AspectJ. It is very useful for safety-critical or realtime systems, but for other kinds of applications, it is not so intuitive to use. In addition, this approach is quite coupled to the base application, since it is necessary to know the state models of system behavior.

\section{CONCLUSIONS}

Current aspect-oriented approaches suffer from well-known problems that rely on the syntactic coupling established between the application and the aspects. A common attempt to give a solution consists of freeing the pointcut definition language from these limitations by describing the join points in a more semantic way.

This paper presents the Blueprint framework, a novel approach to join point identification less coupled to the base-code and providing a finer granularity of selection based on context description. Pointcuts are specified by using patterns (blueprints) of the application expected behavior. More precisely, a join point blueprint is a template on the application expected behavior identifying the join points in their context. In particular join points are captured when the pattern matches portion of the application behavior.

Compared to the current approaches, we can observe some advantages. First of all, we have a more behavioral pointcut definition. In the join point blueprint definition we identify the context of the computational flow we want to match, and the precise point we want to capture. Notwithstanding that, we can still select the join points by using syntactic and structural specification, which is only necessary with a more detailed blueprint. Last but not least, our approach is quite general. It can be applied to every programming language (at the cost of adapting the weaving algorithm to the characteristics of the new language) and used to mimic all the other approaches to AOP. There is also a drawback; the matching phase is quite complex and demands time and space. Fortunately, most of the weaving phase is done once during the compilation and does not affect the performance of the running program.

The Blueprint framework has been completely specified in [20] and a prototype has been implemented. In the future, our plans include improving the prototype, to realize a specific tool to draw the blueprints (at the moment we use Poseidon4UML ${ }^{7}$ ), to organize the statement in class of equivalences for the actions (e.g., $i++$ and $i=i+1$ will be recognized by the same class) and to extend the reflective API. Finally, we want to better check the scalability and robustness of the framework in the software

\footnotetext{
${ }^{7}$ http://www.gentleware.com
} 
evolution context.

\section{ACKNOWLEDGMENTS}

The authors wish to thank Jeff Gray for his help in revising the English of this paper and the anonymous reviewers for their help in improving the paper content with their suggestions.

\section{References}

[1] Walter Cazzola, Jean-Marc Jézéquel, and Awais Rashid. Semantic Join Point Models: Motivations, Notions and Requirements. In Proceedings of SPLAT'06, Bonn, Germany, March 2006.

[2] Walter Cazzola and Sonia Pini. Join Point Patterns: a High-Level Join Point Selection Mechanism. In MoDELS'06 Satellite Events Proceedings, LNCS 4364, pages 17-26, Genova, Italy, October 2006. Springer.

[3] Walter Cazzola, Sonia Pini, and Massimo Ancona. Design-Based Pointcuts Robustness Against Software Evolution. In Proceedings of the 3rd ECOOP Workshop on Reflection, AOP and Meta-Data for Software Evolution (RAMSE'06), pages 35-45, Nantes, France, July 2006.

[4] Rémi Douence, Pascal Fradet, and Mario Südholt. Trace-Based AOP. In Robert E. Filman, Tzilla Elrad, Siobhán Clarke, and Mehmet Akşit, editors, Aspect Oriented Software Development, chapter 9, pages 141-150. AddisonWesley, October 2004.

[5] Rémi Douence and Mario Südholt. A Model and a Tool for Event-Based AspectOriented Programming (EAOP). Technical Report TR 02/11/INFO, École des Mines de Nantes, November 2002.

[6] Robert E. Filman and Daniel P. Friedman. Aspect-Oriented Programming is Quantification and Obliviousness. In Proceedings of OOPSLA 2000 Workshop on Advanced Separation of Concerns, Minneapolis, USA, October 2000.

[7] Kris Gybels and Johan Brichau. Arranging Language Features for More Robust Pattern-Based Crosscuts. In Proceedings of the 2nd Int'l Conf. on Aspect-Oriented Software Development (AOSD'03), pages 60-69, Boston, Massachusetts, April 2003.

[8] Christoph M. Hoffmann and Michael J. O'Donnell. Pattern Matching in Trees. Journal of ACM, 29(1):68-95, 1982.

[9] Wes Isberg. AOP@Work: Check out Library Aspects with AspectJ 5. January 2006. 
[10] Kazunori Kawauchi and Hidehiko Masuhara. Dataflow Pointcut for Integrity Concerns. In Proceedings of the AOSD'04 Workshop on AOSD Technology for Application-level Security, Lancaster, UK, March 2004.

[11] Andy Kellens, Kris Gybels, Johan Brichau, and Kim Mens. A Model-driven Pointcut Language for More Robust Pointcuts. In Proceedings of SPLAT'06, Bonn, Germany, March 2006.

[12] Gregor Kiczales, Erik Hilsdale, Jim Hugunin, Mik Kersten, Jeff Palm, and Bill Griswold. An Overview of AspectJ. In Proceedings of ECOOP'01, pages 327353, Budapest, Hungary, June 2001. ACM Press.

[13] Gregor Kiczales, John Lamping, Anurag Mendhekar, Chris Maeda, Cristina Videira Lopes, Jean-Marc Loingtier, and John Irwin. Aspect-Oriented Programming. In Proceedings of ECOOP'97, LNCS 1241, pages 220-242, Helsinki, Finland, June 1997. Springer-Verlag.

[14] Jacques Klein, Loïc Hélouët, and Jean-Marc Jézéquel. Semantic-based Weaving of Scenarios. In Proceedings of AOSD'06, pages 27-38, Bonn, Germany, March 2006. ACM Press.

[15] Christian Koppen and Maximilian Störzer. PCDiff: Attacking the Fragile Pointcut Problem. In Proceedings of the European Interactive Workshop on Aspects in Software (EIWAS'04), Berlin, Germany, September 2004.

[16] Hsiao-Tsu Lu and Wuu Yang. A Simple Tree Pattern-Matching Algorithm. In Proceedings of the Workshop on Algorithms and Theory of Computation, Chiayi, Taiwan, December 2000.

[17] Noorazean Mohd Ali and Awais Rashid. A State-based Join Point Model for AOP. In Proceedings of the 1st ECOOP Workshop on Views, Aspects and Role (VAR'05), in 19th European Conference on Object-Oriented Programming (ECOOP'05), Glasgow, Scotland, July 2005.

[18] István Nagy, Lodewijk Bergmans, Wilke Havinga, and Mehmet Akşit. Utilizing Design Information in Aspect-Oriented Programming. In Proceedings of 4 th Annual International Conference on Object-Oriented and Internet-based Technologies, Concepts, and Applications for a Networked World (Net.ObjectDays), LNI 61, pages 39-60, Erfurt, Germany, September 2005.

[19] Harold Ossher and Peri Tarr. Hyper/J: Multi-Dimensional Separation of Concerns for Java. In Proceedings of ICSE'01, pages 729-730, Toronto, Ontario, Canada, 2001. IEEE Computer Society.

[20] Sonia Pini. Blueprint: A High-Level Pattern Based AOP Language. PhD thesis, Department of Informatics and Computer Science, Università di Genova, Genoa, Italy, June 2007. 
[21] Awais Rashid and Ana Maria Moreira. Domain Models Are NOT Aspect Free. In Proceedings of MoDELS'06, LNCS 4199, pages 155-169, Genoa, Italy, October 2006. Springer.

[22] J. Alan Robinson. A Machine-Oriented Logic Based on the Resolution Principle. Journal of the ACM, 12(1):23-41, January 1965.

[23] Kouhei Sakurai and Hidehiko Masuhara. Test-based Pointcuts: A Robust Pointcut Mechanism Based on Unit Test Cases for Software Evolution. In Proceedings of Linking Aspect Technology and Evolution revisited (LATE'07), Vancouver, British Columbia, Canada, March 2007.

[24] Dominik Stein, Stefan Hanenberg, and Rainer Unland. Modeling Pointcuts. In Proceedings of the AOSD Workshop on Aspect-Oriented Requirements Engineering and Architecture Design, Lancaster, UK, March 2004.

[25] Tom Tourwé, Andy Kellens, Wim Vanderperren, and Frederik Vannieuwenhuyse. Inductively Generated Pointcuts to Support Refactoring to Aspects. In Proceedings of SPLAT'04, Lancaster, UK, March 2004.

[26] Naoyasu Ubayashi, Genki Moriyama, Hidehiko Masuhara, and Tetsuo Tamai. A Parameterized Interpreter for Modeling Different AOP Mechanisms. In Proceedings of ASE'05, pages 194-203, Long Beach, CA, USA, 2005. ACM Press.

[27] Alexandre Vasseur. Dynamic AOP and Runtime Weaving for Java- How Does AspectWerkz Address It? In Robert E. Filman, Michael Haupt, Katharina Mehner, and Mira Mezini, editors, Proceedings of the 2004 Dynamic Aspect Workshop (DAW'04), pages 135-145, Lancaster, England, March 2004.

\section{ABOUT THE AUTHORS}

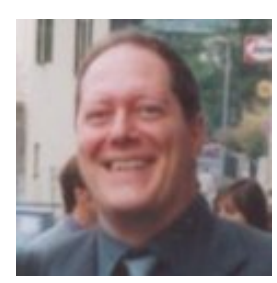

Walter Cazzola (Ph.D.) is currently an assistant professor at the Department of Informatics and Communication (DICo) of the Università degli Studi di Milano, Italy. His research interests include reflection, aspect-oriented programming, programming methodologies and languages. He has written and has served as reviewer of several technical papers about reflection and aspect-oriented programming.

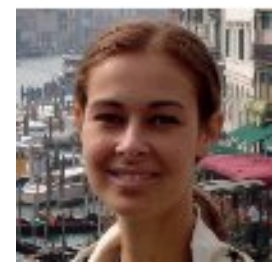

Sonia Pini is a PhD student and research assistant at the Department of Informatics and Computer Science (DISI) of Università degli Studi di Genova, Italy. 